\title{
Kidney function and mortality post-liver transplant in the Model for End-Stage Liver Disease era
}

This article was published in the following Dove Press journal: International Journal of Nephrology and Renovascular Disease 2 November 2011

Number of times this article has been viewed

\author{
Aastha Sethi' \\ Michelle M Estrella' \\ Richard Ugarte ${ }^{2}$ \\ Mohamed G Atta' \\ 'Johns Hopkins University School of \\ Medicine, Department of Medicine, \\ Baltimore, MD, USA; ${ }^{2}$ University \\ of Maryland Medical Center, \\ Department of Medicine, Baltimore, \\ MD, USA
}

\begin{abstract}
The Model for End-Stage Liver Disease (MELD) score incorporates serum creatinine and was introduced to facilitate allocation of orthotopic liver transplantation (LT). The objective is to determine the impact of MELD and kidney function on all-cause mortality. Among LTs performed in a tertiary referral hospital between 1995 and 2009, 419 cases were studied. Cox proportional hazards models were constructed to estimate the hazard ratios (HR) and $95 \%$ confidence intervals (CI) for death. Over mean follow-ups of 8.4 and 3.1 years during the pre-MELD and MELD era, 57 and 63 deaths were observed, respectively. Those transplanted during the MELD era had a higher likelihood of hepatorenal syndrome $(8 \%$ vs $2 \%, P<0.01)$, lower kidney function (median estimated glomerular filtration rate [eGFR] $77.8 \mathrm{vs} 92.6 \mathrm{~mL} /$ $\mathrm{min} / 1.73 \mathrm{~m}^{2}, P<0.01$ ), and more pretransplantation renal replacement therapy (RRT) (5\% vs $1 \% ; P<0.01)$. All-cause mortality risk was similar in the MELD vs the pre-MELD era (HR: 0.98, 95\% CI: $0.58-1.65)$. The risk of death, however, was nearly 3 -fold greater $(95 \% \mathrm{CI}$ : 1.14-6.60) among those requiring pre-transplant RRT. Similarly, eGFR $<60 \mathrm{~mL} / \mathrm{min} / 1.73 \mathrm{~m}^{2}$ post-transplant was associated with a 2.5-fold higher mortality (95\% CI: 1.48-4.11). The study suggests that MELD implementation had no impact on all-cause mortality post-LT. However, the need for pre-transplant RRT and post-transplant kidney dysfunction was associated with a more than 2-fold greater risk of subsequent death.
\end{abstract}

Keywords: eGFR, mortality, MELD, liver transplant

\section{Introduction}

Orthotopic liver transplantation (LT) has emerged as the standard treatment of patients with end-stage liver disease. Since the first successful LT in 1967, more than 100,000 have been performed in the US. ${ }^{1}$ LT offers a reasonable long-term survival benefit, with several centers reporting more than $60 \% 5$-year survival rate after surgery. ${ }^{2-4}$

Due to an increase in the number of patients requiring LT and a relatively stagnant cadaveric donor pool, the number of patients dying on the waiting list has been growing. ${ }^{5}$ To maximize the utility of this scarce resource, the Model for End-Stage Liver Disease (MELD) score was introduced by the United Network for Organ Sharing (UNOS) on February 27, 2002 to facilitate donor liver allocation. The MELD score, calculated from the patient's serum bilirubin, creatinine, and international normalized ratio for prothrombin time (INR), is an objective measurement that predicts the short-term mortality of patients awaiting liver transplantation. It is not affected by "subjective" criteria such as hepatic encephalopathy or ascites. ${ }^{6-10}$ Upon its introduction, however, there was concern that this model may lead to worse outcomes following LT,
Correspondence: Mohamed G Atta Johns Hopkins University School of Medicine, Department of Medicine, I830 E Monument Street, Suite 4I6, Baltimore, MD 21287, USA

$\mathrm{Tel}+\mathrm{I} 4109555268$

$\mathrm{Fax}+\mathrm{I} 4109550485$

Emailmattal@jhmi.edu 
because serum creatinine, a strong negative predictor of short- and long-term post-transplantation survival, was one of the major determinants of a MELD score. ${ }^{11}$ We sought to determine whether implementation of MELD influenced all-cause mortality in the context of perioperative and longterm kidney function among liver transplant individuals using survival analysis.

\section{Methods}

\section{Study design and population}

We conducted a retrospective cohort study using electronic medical records and the UNOS registry. We identified all individuals who underwent LT between May 16, 1995 and April 22, 2009 at a single tertiary hospital with an active liver transplantation program. Individuals who were younger than 18 years, who received multiple organs, who underwent status 1A transplantation, or who had no follow-up 3 months after transplantation were excluded $(n=199)$. Our analysis included 419 individuals. The study was approved by the Johns Hopkins School of Medicine Institutional Review Board.

\section{Outcome}

The primary outcome of interest for this study was all-cause mortality. Vital status was obtained through the National Death Index, electronic medical record, and UNOS registry. Mortality follow-up was completed through April 30, 2010. Time to death was assessed as time from the date of transplantation to the date of death.

\section{Exposures}

The pre-MELD era was defined as the study period before February 27, 2002 when the MELD allocation policy was implemented. The MELD era was defined as the study period on or after this date. During the pre-MELD and MELD era, 163 and 256 individuals, respectively, underwent LT. Pre-transplant variables included demographic factors (age, race, and gender), etiology of liver disease, and comorbid conditions (hepatitis $\mathrm{C}$ infection, diabetes mellitus, and hypertension). Inclusion of diabetes mellitus and hypertension as pre-transplant conditions was based on their presence as documented during the thorough pretransplantation evaluation. The class of drugs used for initial immunosuppression post-LT and length of hospitalization were abstracted from electronic medical records.

Serum creatinine at 1 month before LT was considered as the baseline value. Longitudinal serum creatinine was assessed at 3, 6, 12, and 24 months. Serum creatinine values were carried forward if missing at the next time point. Kidney function was estimated at all of these time points using the chronic kidney disease-epidemiology equation which estimates the glomerular filtration rate (eGFR) based upon age, gender, and race according to serum creatinine level. For each time point, eGFR was dichotomized into $<60 \mathrm{~mL} / \mathrm{min} / 1.73 \mathrm{~m}^{2}$ and $\geq 60 \mathrm{~mL} / \mathrm{min} / 1.73 \mathrm{~m}^{2}$. Patients who underwent at least one session of dialysis during their hospitalization while awaiting LT were designated as having had pre-transplant renal replacement therapy (RRT). Individuals who required RRT immediately following LT were designated as having post-transplant RRT. Hepatorenal syndrome was defined based on clinical diagnosis made by the medical team at the time of a participant's hospitalization.

The MELD score for all individuals who underwent LT in the MELD era was calculated on the day of transplantation in accordance with the UNOS formula: ${ }^{12}$ MELD score $=3.78$ (in serum bilirubin [in $\mathrm{mg} / \mathrm{dL}]$ ) +9.57 (in serum creatinine [in $\mathrm{mg} / \mathrm{dL}]$ ) +11.2 (in INR) +6.43. Serum bilirubin, creatinine, and INR values less than 1 were set to 1 to preclude negative values, and serum creatinine upper-limit values were set at 4.0 if the patient underwent RRT twice within 1 week prior to LT. No adjustments were made for malignancy or others conditions.

\section{Statistical analysis}

Data were analyzed using Stata/MP (v 11.1; StataCorp LP, College Station, TX). Continuous variables were compared using the $t$-test or rank-sum test according to their distribution. Chi-square test was used to compare categorical variables.

Kaplan-Meier product limit estimator function was used to evaluate the time to death following transplant according to the era of LT. Estimates were compared using the log-rank test. Univariable and multivariable Cox proportional hazards models were constructed to estimate the hazard ratio (HR) and 95\% confidence interval (CI) for all-cause mortality between persons who underwent LT during the MELD vs pre-MELD era. Participants were administratively censored on December 31, 2009.

Kidney function was evaluated as a time-varying, binary covariate. For face validity, age, gender, and race were included in the multivariable model regardless of their statistical significance in univariable analyses. The remaining covariates were considered for inclusion in the multivariable model based on their clinical relevance 
and statistical significance. All reported $P$ values were two-sided. Sensitivity analysis, in which observation time was truncated to allow for similar follow-up durations between the pre-MELD and MELD era groups, was also performed. We also performed sensitivity analyses in which individuals with hepatorenal syndrome were excluded.

\section{Results}

The pre-MELD and MELD participants were followed on average for 8.4 and 3.1 years, respectively. Overall mortality was $29 \%$ (57 deaths in pre-MELD era and 63 deaths in MELD era during the follow-up). Table 1 displays sociodemographic and clinical characteristics of patients according to the LT era. Median MELD score for those in MELD era was 19.5 (interquartile range: 14.5-24.0). The most common indication for LT was end-stage liver disease attributed to hepatitis $\mathrm{C}$ infection. Individuals who were transplanted during the MELD

Table I Clinical characteristics according to era of liver transplantation

\begin{tabular}{|c|c|c|c|}
\hline $\begin{array}{l}\text { Clinical } \\
\text { characteristics }\end{array}$ & $\begin{array}{l}\text { Pre-MELD } \\
(n=163)\end{array}$ & $\begin{array}{l}\text { MELD } \\
(n=256)\end{array}$ & $P$ value \\
\hline Age, median year (IQR) & $52(46-58)$ & $53(47-57)$ & 0.41 \\
\hline Black, n (\%) & $33(20)$ & $53(2 I)$ & 0.35 \\
\hline Women, n (\%) & $56(34)$ & $81(32)$ & 0.32 \\
\hline Diabetes, n (\%) & $37(23)$ & $76(3 I)$ & 0.11 \\
\hline Hypertension, n (\%) & $25(16)$ & $60(24)$ & 0.08 \\
\hline \multicolumn{4}{|c|}{ Indication of liver transplantation } \\
\hline Hepatitis C, n (\%) & $60(37)$ & 147 (57) & $<0.01$ \\
\hline Hepatitis B, n (\%) & $4(2)$ & $13(5)$ & \\
\hline Alcohol, n (\%) & $24(15)$ & $22(9)$ & \\
\hline Other, n (\%) & $76(47)$ & $70(27)$ & \\
\hline $\begin{array}{l}\text { Hepatocellular } \\
\text { carcinoma, n (\%) }\end{array}$ & $3(2)$ & $32(12)$ & $<0.0$ I \\
\hline $\begin{array}{l}\text { Hepatorenal } \\
\text { syndrome, n (\%) }\end{array}$ & $4(2)$ & $21(8)$ & 0.02 \\
\hline $\begin{array}{l}\text { Baseline serum creatinine, } \\
\text { median } \mathrm{mg} / \mathrm{dL} \text { (IQR) }\end{array}$ & $0.9(0.7-1.3)$ & I (0.7-I.4) & 0.03 \\
\hline $\begin{array}{l}\text { Baseline eGFR, median } \\
\mathrm{mL} / \mathrm{min} \mid \mathrm{I} .73 \mathrm{~m}^{2}(\mathrm{IQR})\end{array}$ & $\begin{array}{l}92.6 \\
(59.3-111.2)\end{array}$ & $\begin{array}{l}77.7 \\
(51.1-105.2)\end{array}$ & 0.01 \\
\hline \multicolumn{4}{|l|}{ RRT, n (\%) } \\
\hline $\begin{array}{l}\text { Required post-transplant } \\
\text { RRT, n (\%) }\end{array}$ & $8(5)$ & $13(5)$ & 0.57 \\
\hline $\begin{array}{l}\text { Perioperative length } \\
\text { of stay, median days (IQR) }\end{array}$ & $20(12-42)$ & $16(10-30)$ & $<0.01$ \\
\hline \multicolumn{4}{|c|}{ Immunosuppressive medications } \\
\hline Calcineurin inhibitor & 157 (99) & $220(89)$ & $<0.01$ \\
\hline Mycophenolate mofetil & $41(26)$ & $163(74)$ & $<0.01$ \\
\hline Steroids & |4| (89) & $230(94)$ & 0.06 \\
\hline
\end{tabular}

Abbreviations: IQR, interquartile range; eGFR, estimated glomerular filtration rate. era were more likely to have hepatocellular carcinoma (12\% vs $2 \%, P=0.02)$, and suffer from hepatorenal syndrome $(8 \%$ vs $2 \%, P<0.01)$. In addition, participants who underwent LT during the MELD era had significantly lower levels of kidney function (median eGFR 77.8 vs $92.6 \mathrm{~mL} / \mathrm{min} / 1.73 \mathrm{~m}^{2}$, respectively; $P<0.01$ ), with a greater proportion requiring renal replacement therapy prior to transplantation ( $5 \%$ vs $1 \% ; P<0.01)$. The initial immunosuppressive regimens varied between the two groups, with a greater proportion of individuals transplanted during the MELD era receiving mycophenolate mofetil $(74 \%$ vs $26 \% ; P<0.01)$ and fewer of these receiving calcineurin inhibitors $(89 \%$ vs $99 \%$; $P<0.01)$.

\section{All-cause mortality}

Kaplan-Meier curves showed that patients transplanted during the MELD era had survival rates similar to those transplanted in the pre-MELD era $(P=0.14$; Figure 1$)$. The risk of all-cause death associated with LT in the MELD vs the pre-MELD era was similar in the unadjusted and adjusted analyses (crude HR: 1.35, 95\% CI: 0.91-2.00; adjusted HR: $0.98,95 \%$ CI: $0.58-1.65$; Table 2). However, receipt of pre-transplant RRT was associated with significantly greater risk for all-cause mortality (Figure 2). In adjusted analyses, the risk of death was nearly 3 -fold greater $(95 \% \mathrm{CI}$ : 1.14-6.60) among individuals who required pre-transplant RRT. Similarly, the HR for death was 2.5-fold higher (95\% CI: 1.48-4.11) among persons who had eGFR $<60 \mathrm{~mL} /$ $\mathrm{min} / 1.73 \mathrm{~m}^{2}$ post-transplant. These risk estimates were unchanged when follow-up time was truncated to allow similar follow-up times between the pre-MELD and MELD era groups. Adjusted models excluding individuals with hepatorenal syndrome yielded similar risk estimates, but

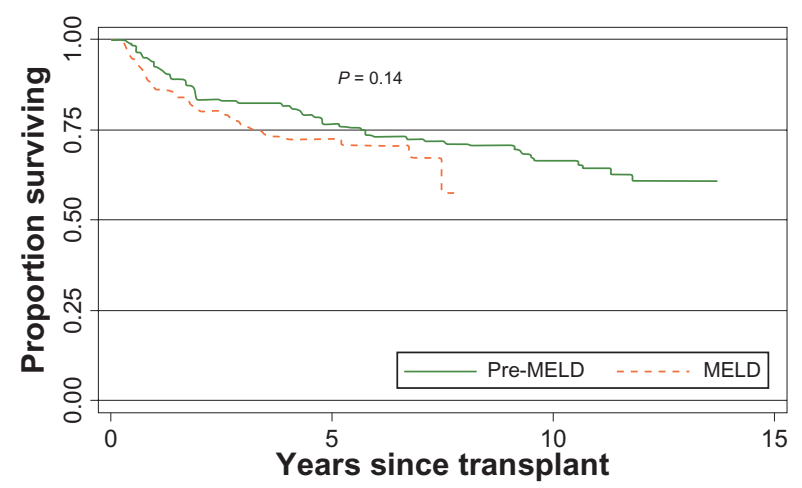

Figure I Kaplan-Meier survival estimates by MELD era with 120 (28.6\%) deaths observed. Estimates similar in sensitivity analyses. 
Table 2 Cox proportional hazards models for all-cause mortality

\begin{tabular}{|c|c|c|c|c|}
\hline \multirow[t]{2}{*}{ Covariates } & \multicolumn{2}{|l|}{ Univariable } & \multicolumn{2}{|c|}{ Multivariable $(n=394)$} \\
\hline & HR $(95 \% \mathrm{Cl})$ & $P$ value & HR $(95 \% \mathrm{Cl})$ & $P$ value \\
\hline MELD era & $1.35(0.9 \mathrm{I}-2.00)$ & 0.14 & $0.98(0.58-1.65)$ & 0.94 \\
\hline Female & $0.92(0.63-1.36)$ & 0.70 & $1.03(0.62-1.70)$ & 0.91 \\
\hline Black & $1.00(0.74-1.33)$ & 1.00 & $1.15(0.81-1.65)$ & 0.43 \\
\hline Age, per year older & $1.00(0.98-1.02)$ & 0.89 & $0.99(0.97-1.02)$ & 0.68 \\
\hline Required RRT pre-transplant & $2.46(1.14-5.28)$ & 0.02 & $2.75(1.14-6.60)$ & 0.02 \\
\hline Hepatitis $C$ seropositive & $1.56(1.09-2.24)$ & 0.02 & $1.56(0.96-2.54)$ & 0.07 \\
\hline Hypertension & $0.97(0.89-1.54)$ & 0.90 & $1.27(0.69-2.32)$ & 0.44 \\
\hline Diabetes & $0.89(0.58-1.36)$ & 0.59 & $0.57(0.31-1.04)$ & 0.07 \\
\hline Time-varying eGFR status ( $<60$ vs $\geq 60)^{*}$ & $2.17(1.37-3.43)$ & $<0.01$ & $2.47(1.48-4.11)$ & $<0.01$ \\
\hline
\end{tabular}

Note: *ln $\mathrm{mL} / \mathrm{min} / \mathrm{l} .73 \mathrm{~m}^{2}$.

Abbreviations: eGFR, estimated glomerular filtration rate; MELD, Model for End-Stage Liver Disease; RRT, renal replacement therapy.

the hazard of death associated with pre-transplant RRT no longer reached statistical significance (adjusted HR: 2.98, 95\% CI: 0.90-9.87).

\section{Discussion}

The risk of all-cause mortality remains high, but is similar between the MELD and pre-MELD era. The need for pretransplant RRT, and kidney dysfunction after transplantation, are associated with a more than 2-fold greater risk of death after LT.

Similar to our findings, Yoo and Thuluvath also found that the post-liver transplant survival was unaffected in patients in whom the MELD score was used for organ prioritization. However, their follow-up was relatively short ( $<10$ months). ${ }^{13}$ Although one may argue that comparable outcomes between the MELD and pre-MELD era could be explained by the high rate of hepatorenal syndrome in the MELD era, which is likely to improve upon LT, our findings were not affected by excluding those patients from the analyses. While the MELD score attempts to capture the

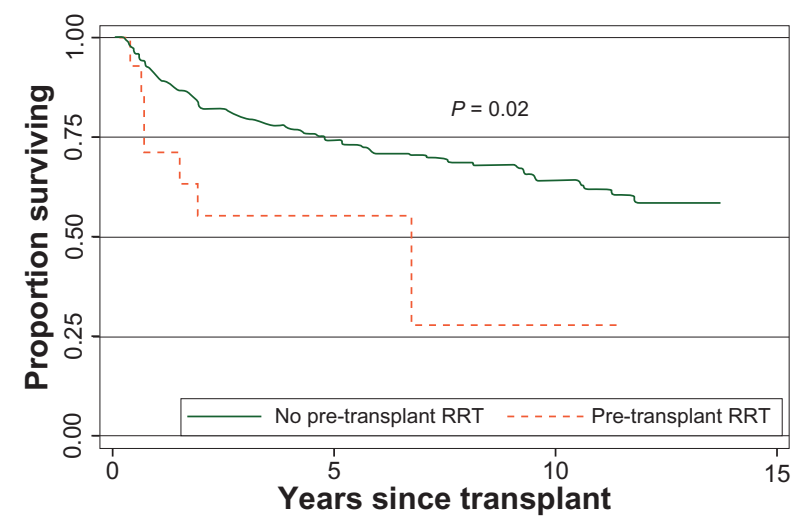

Figure $\mathbf{2}$ Kaplan-Meier survival estimates by receipt of renal replacement therapy before liver transplant. severity of liver disease among potential liver transplant recipients, the majority of its components include parameters such as serum bilirubin and INR values, which normalize upon transplantation of a functional liver. Moreover, the initial purpose of the MELD score was to predict death shortly following transjugular intrahepatic portosystemic shunt ${ }^{14}$ and subsequently to prognosticate outcomes among individuals with end-stage liver disease. ${ }^{8}$ Conversely, many factors aside from the use of MELD scores such as advances in surgical techniques, immunosuppression, anti-viral therapy, and most importantly, severity of underlying illness, may have influenced outcomes. If MELD has resulted in the transplantation of sicker patients as widely acknowledged, then comparable mortality rates in sicker patients suggest better outcomes rather than equal outcomes. The study by Bencker et al documented that despite the significant increase in the calculated MELD score in the post-MELD era compared to the pre-MELD era, 1-year survival post-transplant was not different. ${ }^{15}$ Those results confirm that this model was not developed to evaluate outcomes after liver transplant but rather to capture the severity of liver disease.

Similar to prior studies, we observed a significant risk of death associated with pre-transplant RRT and kidney dysfunction post-LT. Nair and colleagues demonstrated that pre-transplant renal dysfunction was an independent predictor of 30-day and 2-year mortality after adjusting for the recipient's age, sex, etiology of liver disease, diabetes status, body mass index, cold ischemic time, and UNOS status. ${ }^{11}$ In their study, a creatinine clearance of less than $40 \mathrm{~mL} / \mathrm{min}$ was associated with significantly lower shortand long-term graft and patient survival rates. ${ }^{11}$ Similarly, pre-operative renal dysfunction was also an independent predictor of both short- and long-term survival after LT in 
a small number of patients with non-biliary cirrhosis and fulminant hepatic failure and cirrhosis. ${ }^{16-19}$

Few studies have evaluated the relationship between the need for RRT post-transplant and overall mortality. Afonso and colleagues analyzed data collected prospectively from 152 consecutive LTs performed by the same team from March 2003 to November 2007. ${ }^{20}$ They observed that patients who developed severe renal failure post-LT (serum creatinine $>3 \mathrm{mg} / \mathrm{dL}$ or needing RRT) had worse outcomes compared with other patients; $95.29 \%$ vs $69.69 \%$ and $86.95 \%$ vs $41.66 \%$ for early and 1 -year survivals, respectively $(P<0.001) .{ }^{20}$ However, follow-up was shorter and the study included fewer patients compared with our study. In addition, our study is important as it does highlight the association of both pre-transplant and post-transplant renal failure with overall mortality.

Our study has several limitations to consider. We did not calculate the MELD score and thus the clinical risk profile for participants transplanted in the pre-MELD era may have differed from those in the MELD era. We, however, adjusted for one of the main components in the MELD calculation, serum creatinine. We did not have sufficient comparative data on pretransplant and intraoperative factors such as donor characteristics, ischemia time, and intraoperative blood loss. These factors, however, are likely to impact the peri operative mortality rather than mortality beyond 90 days post-transplant. Finally, significant differences in immunosuppressive regimens existed between the pre-MELD and MELD eras for which we could not adjust due to collinearity. As the indication for use of mycophenolate mofetil vs other immunosuppressive drugs is unknown, its association with renal function and therefore mortality is unclear. Although mycophenolate mofetil is non-nephrotoxic, patients who had underlying kidney disease may have been channeled to this drug.

Despite early concerns that implementation of the MELD allocation policy would augment the risk for death among liver recipients, our study demonstrates that individuals transplanted in the MELD era experience similar survival as those transplanted in the pre-MELD era. Pre-transplant RRT and kidney function impairment following LT appears to be significantly associated with increased risk of death. Our findings outline the importance of monitoring kidney function among LT recipients. Further studies are needed to elucidate the mechanisms by which kidney disease may contribute to excess mortality in this patient population.

\section{Acknowledgments}

Michelle M Estrella is supported by the NIH-NIDDK grant K23DK081317.

\section{Disclosure}

The authors report no conflicts of interest in this work.

\section{References}

1. US Transplants Performed 1988-2011. Available from: http:// optn.transplant.hrsa.gov/latestData/rptData.asp. Accessed April 10, 2011.

2. Bismuth H, Majno PE, Adam R. Liver transplantation for hepatocellular carcinoma. Semin Liver Dis. 1999;19(3):311-322.

3. Jain A, Reyes J, Kashyap R, et al. Long-term survival after liver transplantation in 4,000 consecutive patients at a single center. Ann Surg. 2000;232(4):490-500.

4. Jonas S, Bechstein WO, Steinmuller T, et al. Vascular invasion and histopathologic grading determine outcome after liver transplantation for hepatocellular carcinoma in cirrhosis. Hepatology. 2001;33(5): $1080-1086$.

5. 2009 Annual Report of the US Organ Procurement and Transplantation Network and the Scientific Registry of Transplant Recipients: Transplant Data 1999-2008. US Department of Health and Human Services, Health Resources and Services Administration, Healthcare Systems Bureau, Division of Transplantation, Rockville, MD. Available from: http://www.ustransplant.org/annual_reports/current/default.htm. Accessed April 11, 2011.

6. Botta F, Giannini E, Romagnoli P, et al. MELD scoring system is useful for predicting prognosis in patients with liver cirrhosis and is correlated with residual liver function: a European study. Gut. 2003;52(1):134-139.

7. Freeman RB Jr, Wiesner RH, Harper A, et al. The new liver allocation system: moving toward evidence-based transplantation policy. Liver Transpl. 2002;8(9):851-858.

8. Kamath PS, Wiesner RH, Malinchoc M, et al. A model to predict survival in patients with end-stage liver disease. Hepatology. 2001;33(2): 464-470.

9. Wiesner R, Edwards E, Freeman R, et al. Model for end-stage liver disease (MELD) and allocation of donor livers. Gastroenterology. 2003;124(1):91-96.

10. Wiesner RH, McDiarmid SV, Kamath PS, et al. MELD and PELD: application of survival models to liver allocation. Liver Transpl. 2001 7(7):567-580.

11. Nair S, Verma S, Thuluvath PJ. Pretransplant renal function predicts survival in patients undergoing orthotopic liver transplantation. Hepatology. 2002;35(5):1179-1185.

12. Organ Distribution: Allocation of Livers. Available from: http://optn. transplant.hrsa.gov/PoliciesandBylaws2/policies/pdfs/Policy_8.pdf. Accessed April 10, 2011.

13. Yoo HY, Thuluvath PJ. Short-term postliver transplant survival after the introduction of MELD scores for organ allocation in the United States. Liver Int. 2005;25(3):536-541.

14. Malinchoc M, Kamath PS, Gordon FD, Peine CJ, Rank J, ter Borg PC. A model to predict poor survival in patients undergoing transjugular intrahepatic portosystemic shunts. Hepatology. 2000;31(4): 864-871.

15. Benckert C, Quante M, Thelen A, et al. Impact of the MELD allocation after its implementation in liver transplantation. Scand J Gastroenterol. 2011;46(7-8):941-948.

16. Brown RS Jr, Lombardero M, Lake JR. Outcome of patients with renal insufficiency undergoing liver or liver-kidney transplantation. Transplantation. 1996;62(12):1788-1793. 
17. Gonzalez E, Rimola A, Navasa M, et al. Liver transplantation in patients with non-biliary cirrhosis: prognostic value of preoperative factors. J Hepatol. 1998;28(2):320-328.

18. Lafayette RA, Pare G, Schmid CH, King AJ, Rohrer RJ, Nasraway SA. Pretransplant renal dysfunction predicts poorer outcome in liver transplantation. Clin Nephrol. 1997;48(3):159-164.
19. Mendoza A, Fernandez F, Mutimer DJ. Liver transplantation for fulminant hepatic failure: importance of renal failure. Transpl Int. 1997; 10(1):55-60.

20. Afonso RC, Hidalgo R, Zurstrassen MP, et al. Impact of renal failure on liver transplantation survival. Transplant Proc. 2008;40(3): $808-810$.

\section{Publish your work in this journal}

The International Journal of Nephrology and Renovascular Disease is an international, peer-reviewed open-access journal focusing on the pathophysiology of the kidney and vascular supply. Epidemiology, screening, diagnosis, and treatment interventions are covered as well as basic science, biochemical and immunological studies. The journal welcomes original research, clinical studies, reviews \& evaluations, expert opinion and commentary, case reports and extended reports. The manuscript management system is completely online and includes a very quick and fair peerreview system, which is all easy to use. Visit http://www.dovepress.com/ testimonials.php to read real quotes from published authors.

Submit your manuscript here: http://www.dovepress.com/international-journal-of-nephrology-and-renovascular-disease-journal 